\title{
Alter
}

Revue de phénoménologie

$21 \mid 2013$

La Vie

\section{Vie et différences anthropologiques}

\section{Julien Pieron}

\section{OpenEdition}

\section{Journals}

Édition électronique

URL : http://journals.openedition.org/alter/826

DOI : $10.4000 /$ alter.826

ISSN : 2558-7927

\section{Éditeur :}

Association ALTER, Archives Husserl (CNRS-UMR 8547)

\section{Édition imprimée}

Date de publication : 1 novembre 2013

Pagination : 135-149

ISBN : 978-2-95-223749-9

ISSN : 1249-8947

\section{Référence électronique}

Julien Pieron, «Vie et différences anthropologiques », Alter [En ligne], 21 | 2013, mis en ligne le 01 juin 2019, consulté le 05 juillet 2019. URL : http://journals.openedition.org/alter/826 ; DOI : 10.4000/ alter.826 


\title{
VIE ET DIFFÉRENCES ANTHROPOLOGIQUES
}

\author{
Julien Pieron
}

J'ai peur que l'on ne comprenne pas bien ce que j'entends par issue. J'emploie le mot dans son sens courant et dans toute son amplitude. J'évite intentionnellement de parler de liberté. Ce n'est pas ce grand sentiment de la liberté dans tous les sens auquel je songe. Comme singe je le connaissais peut-être, et j'ai vu des hommes qui en éprouvent le désir. Mais, en ce qui me concerne, je n'ai jamais réclamé ni ne réclame la liberté.

(Kafka)

Les pages qui suivent tentent de dresser le bilan de recherches déjà anciennes autour de la phénoménologie de la vie et du problème de la différence anthropologique. Ces recherches trouvent leur point de départ dans une lecture minutieuse de Heidegger, et débouchent sur une série de doutes et de difficultés, qui conduisent à aller voir et chercher ailleurs qu'en phénoménologie - notamment du côté de la philosophie politique et de l'épistémologie. Après avoir rappelé brièvement la source de ces doutes et difficultés, nous tenterons d'y répondre de la seule façon qui nous apparaisse aujourd'hui possible : interroger et déplacer radicalement les conditions du problème, reformuler intégralement les questions - d'où le nombre et le caractère hétéroclite (ou hétérodoxe) des auteurs invoqués ici.

Le cadre de nos recherches autour de la différence anthropologique, c'est une étude de l'interprétation de la Monadologie leibnizienne présentée par Heidegger dans son cours du semestre d'été 1928. Cette étude permettait d'atteindre un double résultat: confirmer une interprétation de Sein und Zeit qui visait à reconstruire la troisième section «Temps et être », ou du moins à cerner le mouvement qui s'y accomplit; offrir un schème conceptuel susceptible d'éclairer la genèse de la conceptualité du cours de 1929-30 et de mettre en évidence une sorte de carrefour métaphysique entre une 
voie empruntée et creusée historiquement par Heidegger, et une autre voie, toute virtuelle et seulement à l'état de friche.

$\mathrm{Qu}$ 'est-ce donc qui se joue dans l'interprétation heideggérienne de la Monadologie? D'abord une projection de la conceptualité de Sein und Zeit dans le texte leibnizien, aussi lourde de conséquences que celle que Heidegger effectue à la même période dans le texte de Kant. Ce que révèle cette projection, c'est la thématique de l'identité de l'être de l'étant privilégié et de l'être en général, qui permet le renversement $d^{\prime}$ " être et temps » en " temps et être », ou encore le passage de la problématique de la temporalité [Zeitlichkeit] du Dasein à celle de la Temporalité [Temporalität] de l'être. Ce qui s'impose également, si on prend le temps de retraduire en vocabulaire phénoménologique la traduction ou le transfert de Heidegger en Leibniz, c'est la nécessité de poser une question nouvelle : celle de l'origine de la différence, plus précisément celle de la production immanente de la différence ${ }^{1}$ au sein même de cette identité - question qui appelle une reprise du problème métaphysique de l'individuation ou de la subjectivation. Ce qui se joue ensuite dans l'interprétation heideggérienne de la monadologie, dans cette projection de Sein und Zeit dans le lexique métaphysique leibnizien, $c^{\prime}$ est une sorte d'infléchissement «vitaliste » et pulsionnel de la conceptualité d'Être et temps, qui constitue la matrice ou le laboratoire de la plupart des notions qui vont servir à Heidegger pour penser, de façon « privative », l'animalité dans son cours de 1929-30².

En transposant les traits fondamentaux de l'analytique du Dasein à la monade caractérisée comme Drang (élan, poussée, pulsion), Heidegger ouvrait également la porte à une extension « vitaliste » de l'ontologie phénoménologique, extension dans laquelle les foyers finis d'ouverture de l'être ne se limiteraient plus au seul domaine de l'humanité, mais engloberaient toutes les formes possibles de vie. C'est en ce sens que nous avons proposé de considérer le cours de 1928 comme une sorte de carrefour métaphysique entre deux voies possibles: ou bien celle d'une restriction des foyers d'ouverture de l'être au seul domaine de l'humanité - restriction qui s'effectuera dans le cours de 1929-30 via la thèse de la pauvreté en monde d'un animal dénué du rapport à l'étant comme tel, et qui débouchera sur la thématique de l'histoire de l'être comme «histoire essentielle de

\footnotetext{
${ }^{1}$ Différence ontologique entre être et étant, différence noétique entre être en général et essence de l'homme. Cf. Julien Pieron, Pour une lecture systématique de Heidegger, Bruxelles, Ousia, 2010.

${ }^{2}$ Cf. sur ce point Julien Pieron, «De l'analytique existentiale à la zoologie privative : le problème de la différence anthropologique et l'amorce du "tournant" », Alter no 17, 2009, p. 195-212.
} 
$l^{\prime}$ homme $»^{3}-$, ou bien celle d'une multiplication des foyers vivants (et non seulement existants) d'ouverture de l'être, qui aurait exigé la construction d'une ontologie phénoménologique à foyers multiples suivant le schème $d$ 'une "monadologie phénoménologique » dans laquelle chaque monade déploie le monde ou l'univers suivant un point de vue fini qui la singularise 4 .

On sait que Heidegger envisage en bloc l'ensemble des étants qu'il regroupe sous la catégorie d'animal, au singulier collectif : «l'animal ». Ce geste, amplement discuté par Derrida dans plusieurs de ses textes, soulève plusieurs difficultés, dont la principale concerne peut-être la façon dont le phénoménologue utilise les sciences empiriques de son temps (embryologie, biologie, éthologie naissante). L'utilisation des savoirs empiriques contemporains, non comme une simple illustration, mais comme un véritable guide pour le travail philosophique, constitue un hapax dans l'œuvre de Heidegger, et l'un des traits qui rendent ce cours absolument passionnant. Elle ouvre aussi la porte à une série d'interrogations « critiques » qui peuvent nous faire dériver et nous emmener ailleurs.

En posant a priori l'unité d'un domaine, «l'animal », qu'il oppose massivement et radicalement à l'homme, Heidegger se donne la possibilité - peut-être un peu trop facile ou rapide - d'utiliser n'importe quel exemple biologique ou éthologique comme témoignant de manière fiable de l'ensemble du domaine que constitue «l'animal ». On notera ainsi que de nombreuses analyses du cours de 1929-30 s'appuient sur des exemples empruntés à l'entomologie, ou à l'étude des unicellulaires, et très peu - voire aucune - sur des exemples tirés du champ de la primatologie. Cette démarche n'est pas sans conséquences, dans la mesure où, s'attardant à dessein sur des êtres qui nous offrent des possibilités extrêmement réduites d'accompagnement, elle favorise et conforte à peu de frais l'impression d'une différence abyssale entre l'homme et «l'animal » dans sa plus grande généralité.

Cette partialité dans le choix des exemples n'est cependant pas le fruit d'une ruse ou d'une volonté de duper: elle est réfléchie et fondée philosophiquement. L'argument utilisé à plusieurs reprises par Heidegger pour justifier le choix de tels exemples, c'est le souci d'éviter l'anthropomorphisme. Souci critique peut-être louable, mais dont on pourrait interroger l'histoire, et se demander s'il n'est tout de

\footnotetext{
${ }^{3}$ Heidegger, GA 10, Der Satz vom Grund, Vittorio Klostermann, Francfort-sur-le-Main, 1997, p. 139.

${ }^{4}$ On retrouve dans ce dernier programme la suite des trois concepts fondamentaux que sont le monde, la finitude, et la solitude ou l'individuation.
} 
même pas lié à la position (ou à la pétition) de principe suivant laquelle l'homme diffère radicalement de l'animal. Cette position n'est en effet pas le résultat d'une enquête empirique, mais une sorte d'a priori qui guide le travail philosophique (et sans doute aussi l'expérience quotidienne), a priori qu'aucun fait empirique ne pourra jamais remettre en question. Dans une telle situation, la critique de l'anthropomorphisme pourrait constituer une façon d'écarter à relativement peu de frais, et non sans une certaine mauvaise foi, les faits empiriques - ou les interprétations de faits empiriques - qui risqueraient d'inquiéter l'a priori ou la ligne de démarcation radicale tracée entre l'homme et «l'animal ».

En laissant de côté le soupçon de mauvaise foi, il semble qu'à partir du moment où l'on présuppose une différence radicale entre l'homme et «l'animal », on doive nécessairement qualifier d'anthropomorphique (au sens d'attribuant indûment à «l'animal» ce qui constitue un propre de l'homme) toute description empirique de comportements animaux évoquant un peu trop les comportements humains - en ce qu'ils comporteraient par exemple l'idée d'invention, de plaisir «désintéressé » ou de "gratuité » biologique, toutes caractéristiques généralement conférées aux comportements humains et à eux seuls. Dans plusieurs de ses travaux ${ }^{5}$, Vinciane Despret insiste sur le fait que le reproche d'anthropomorphisme a une histoire, qui coïncide avec celle de la constitution de l'éthologie comme science, et la conquête d'un territoire disciplinaire ou académique qui s'établit aussi par la dévalorisation du savoir " profane » des naturalistes amateurs, des éleveurs, des dresseurs, des chasseurs, des gardiens de zoo, etc.

La conséquence de cette façon de faire science, c'est par exemple que le registre de l'anecdote, encore considéré comme légitime par Darwin lui-même, sera banni du domaine de la science éthologique, et qu'un ensemble de concepts ou de thèmes de recherches vont être progressivement tenus à distance par des chercheurs qui s'autocensurent, de crainte d'essuyer le refus des comités de lecture ou des institutions qui financent leurs recherches. Parmi ces thèmes et ces concepts, on trouve notamment la question du plaisir et du bien-être. Ainsi c'est seulement depuis une époque récente qu'il est devenu possible, en éthologie, d'avancer sans griller à coup sûr sa carrière académique l'hypothèse qu'un oiseau puisse chanter aussi «pour le

\footnotetext{
${ }^{5}$ Dont le récent Que diraient les animaux, si... on leur posait les bonnes questions?, Paris, Les empêcheurs de penser en rond/La découverte, 2012.
} 
plaisir ». Il faut néanmoins ajouter que ce genre de générosité herméneutique est loin d'être la règle.

Nous illustrerons brièvement cette situation par un cas, celui d'un échange de courriels plutôt houleux entre éthologues et anthropologues, au sujet de l'interprétation d'une vidéo postée par une famille russe sur youtube. Cette vidéo (http://www.youtube.com/watch?v= dR0Ptvpw66E) montre une corneille qui, posée sur un objet rond et plat, semble s'amuser - c'est en tout cas le premier sentiment du spectateur non prévenu - à glisser, encore et encore, le long d'un toit enneigé ${ }^{6}$. À l'évidence, l'éthologue cognitiviste qui fait circuler cette vidéo du «corbeau skieur» parmi ses collègues est partisan d'une interprétation « généreuse » de ce comportement, y voyant une forme $\mathrm{d}$ 'invention ludique et de plaisir. Plusieurs de ses collègues la rappellent pourtant immédiatement à l'ordre, et relisent le même comportement dans des termes purement fonctionnels et utilitaires - sans aucune "gratuité »-, en ne faisant intervenir qu'une conjugaison de schèmes comportementaux innés et de circonstances parfaitement objectives (becquetage, toit verglacé, etc.). On notera que les deux interprétations semblent également plausibles, mais que lire l'interprétation «fonctionnaliste » avant de visionner la vidéo risque de modifier fortement la façon dont nous recevons ces images, et le rapport que nous pourrions tisser à l'oiseau.

L'évocation de cette micro-controverse, qui pose le problème de ce qui peut ou non se dire quand on veut faire science, retrouve un thème abordé par Heidegger dans son cours de $1929-30^{7}$ : celui de la dimension performative du langage et de la nomination, qui nous offre l'occasion de penser et de vivre ou d'exister autrement. C'est à l'occasion d'une discussion critique de la problématique de l'Einfühlung que Heidegger avance l'idée qu'un changement de langue et de conceptualité pourrait constituer en soi une expérience et une véritable transformation de l'existence :

Ce n'est pas par entêtement ni par souci d'originalité en philosophie que nous ne parlons plus aujourd'hui d'expériences vécues, ni d'expériences vécues dans la conscience, ni de conscience : nous sommes contraints à un autre langage en raison d'une transformation de l'existence. Plus précisément, ce changement a lieu avec cet autre langage. Si nous abandonnons aujourd'hui le mot "intropathie », que nous choisissons de parler (même de façon provisoire et sous réserve) de transposition dans

\footnotetext{
${ }^{6}$ Je remercie Vinciane Despret de m'avoir fait découvrir cette micro-controverse éthologique.

${ }^{7}$ Heidegger, GA 29/30, Die Grundbegriffe der Metaphysik, Welt - Endlichkeit - Einsamkeit, Vittorio Klostermann, Francfort-sur-le-Main, 2004³ ; Les concepts fondamentaux de la métaphysique, Mondefinitude - solitude, Paris, Gallimard, 1992.
} 
l'autre, et qu'ainsi nous parlons autrement, il ne s'agit pas, dans notre cas, du choix d'une meilleure expression, qui exprimerait la même chose: c'est l'idée et la chose qui sont devenues autres (GA 29/30, trad. fr., p. 301).

On trouve dans cette remarque jetée en passant l'esquisse d'un motif central de la pensée du second Heidegger: l'idée que la nomination participe à l'avènement de nouveaux rapports à l'être. Cette remarque touche à un problème central de toute approche de la question de l'animalité : celui de la langue qu'on emploie pour décrire ou pour parler de "l'animal», celui de la compréhension d'être et des a priori (perceptifs, relationnels, ontologiques) qui sont véhiculés par la langue. Dans un passage où il s'intéresse aux animaux domestiques, Heidegger note que la langue allemande emploie deux verbes distincts (essen/fressen) pour désigner l'acte de se nourrir selon qu'il est humain ou animal ${ }^{8}$. On trouverait sans peine en français des doublets (par ex. visage/gueule) qui véhiculent le même type de distinction. C'est donc ici dans la langue maternelle elle-même que réside la précompréhension d'une différence radicale entre l'homme et l'animal.

Il n'est pourtant pas dit que la langue maternelle et la compréhension d'être qu'elle charrie soit un absolu, et que des expériences ou expérimentations semblables à celle que Heidegger propose en tentant de dépasser le questionnement en terme d'Einfühlung ne soient pas possibles, qui participent elles aussi à un bouleversement de l'existence et à une transformation de nos rapports aux vivants non-humains. C'est dans cette brèche, ouverte par l'idée d'une dimension performative de la langue, que s'engagent certains des penseurs actuels les plus intéressants de la question animale. On pourrait évoquer les travaux de Donna Haraway, chez qui la dimension d'une écriture volontiers provocatrice, à la fois tendre et impudique, est si importante, parce qu'elle vise - un peu sur le modèle des mobilisations queer - à sortir tel ou tel animal du placard, à présenter des faits (par ex., à la fin du Companion Species Manifesto, la sexualité - débridée, non fonctionnelle, non reproductive - de sa chienne Cayenne) et des façons de parler qui n'avaient jusqu'ici pas droit de cité dans le monde philosophique et scientifique académique.

On mentionnera également la proposition que la sociologue Jocelyne Porcher, elle-même ancienne éleveuse, fait aux éleveurs de considérer que leurs animaux travaillent eux aussi, et travaillent avec

${ }^{8}$ GA 29/30, p. 308 (trad. p. 310). 
les humains. Cette proposition est à la base d'une observation quasi sociologique des animaux d'élevage, particulièrement attentive aux disfonctionnements, aux moments où les vaches cessent en quelque sorte de coopérer et « d'y mettre du leur »- ce qui permet de révéler par contraste l'existence d'une véritable attention et implication animales, d'un engagement et d'une intelligence sociale ou relationnelle passant complètement inaperçus dans les moments où « tout va bien ». Face à une telle proposition, on sera peut-être d'abord tenté de crier à l'anthropomorphisme ; il est moins sûr qu'on puisse le faire si on prend le temps de lire les livres ou d'écouter les conférences de Jocelyne Porcher, dont la finesse et l'intelligence scientifique forcent le respect, et qui nous donnent accès à un monde incroyablement peuplé et intéressant. On notera aussi que les recherches de Porcher débouchent sur - ou sont nourries par? - une prise de position politique, qui n'a pas le simplisme de l'idée de « libération animale », mais qui s'articule au contraire autour d'une défense de la pratique (qui est aussi un mode vie) de l'élevage, une défense nuancée, focalisée sur la qualité des relations et des formes d'être-avec, et sur leurs conditions socio-économiques de possibilité ou d'impossibilité.

Passons maintenant à la présentation d'un doute ou d'une aporie concernant l'idée d'ontologie phénoménologique à foyers multiples. Au fond, le projet d'une ontologie à foyers multiples suppose de s'engager dans la question de savoir « ce que ça fait »d'être, non pas un animal en général, mais tel ou tel animal en particulier, d'en explorer en quelque sorte le monde et l'expérience en première personne. Dans la mesure où cette expérience en première personne nous est inaccessible, il semble qu'on ne puisse que se réduire à un pis-aller : reconstruire, à partir des données empiriques de l'éthologie ou de la psychologie animale, un analogon de cette expérience animale particulière, et du monde qui y est ouvert. Et c'est d'une certaine façon ce que Heidegger tente dans son cours en s'inspirant notamment des travaux d'Uexküll - pour aboutir à la conclusion que l'animal est privé du rapport à l'étant comme tel, exclu de la manifesteté de l'étant, et qu'il est donc vain de vouloir ancrer une ontologie dans cette ouverture qui est tout aussitôt et tout aussi bien une fermeture.

Nous avons suggéré ailleurs ${ }^{9}$ que cette conclusion n'est pas un forçage de la part de Heidegger : elle découle rigoureusement de la

\footnotetext{
${ }^{9}$ Cf. Julien Pieron, «Monadologie et/ou constructivisme ? Heidegger, Deleuze, Uexküll», Bulletin d'analyse phénoménologique VI 2, 2010 (Actes 2), p. 86-117 [http://popups.ulg.ac.be/bap/ document.php?id=384].
} 
théorie et des descriptions d'Uexküll ${ }^{10}$. Si l'on prend en effet au sérieux la théorie uexküllienne du «cercle fonctionnel»- au sein duquel s'enchaînent "caractères perceptifs » et "caractères actifs » des objets, qui s'éteignent et se relancent mutuellement, chaque facteur signifiant du monde ambiant suscitant dans le chef de l'animal-sujet une réponse fonctionnelle ou un comportement parfaitement ajusté ou adapté, qui vient en quelque sorte neutraliser ou éteindre ce caractère signifiant, - il semble que les conclusions heideggériennes au sujet de l'accaparement comme privation de la saisie de quelque chose comme quelque chose s'imposent. À lire les descriptions d'Uexküll, on a peut-être affaire à un animal-sujet, mais ce sujet a tout d'un somnambule, un somnambule absorbé dans son monde, un somnambule dont les réactions s'accordent si parfaitement et si fonctionnellement aux caractères signifiants de son milieu qu'on doute que ce milieu puisse acquérir une véritable épaisseur ou présence objective.

On pourrait montrer que l'idée de perfection du comportement animal, l'idée d'un mouvement qui s'épuise dans une pure fonctionnalité, l'idée d'une adaptation et d'une intégration sans reste est commune à Uexküll et Heidegger, et qu'elle surgit, dans le cours de 1929-30, bien avant la description du comportement animal. C'est en effet d'abord au niveau somatique, à la faveur d'une description phénoménologique de l'organe, qu'apparaît la thématique d'un êtreau-service de la pulsion, d'une soumission parfaite et sans reste. C'est cette idée de perfection animale (adaptation parfaite, soumission sans reste aux nécessités pulsionnelles/fonctionnelles) qui fait que Heidegger comme Uexküll sont incapables de faire droit à la théorie de l'évolution: si la possibilité d'un décrochage, d'une monstruosité ou d'un raté de la fonctionnalité, d'une latitude perceptive et comportementale, n'est pas inscrite d'une façon ou d'une autre dans les formes qu'on jugera les plus élémentaires de la vie, on ne pourra en effet jamais les retrouver à l'arrivée, ni rendre compte de la possibilité de l'évolution, et a fortiori du mystère que constituerait dans ces coordonnées - le passage de «l'animal » à l'homme.

Sur ce point, comme sur beaucoup d'autres, il conviendrait de jouer Canguilhem contre Uexküll et Heidegger. Malgré leur critique proclamée du mécanisme, Uexküll et Heidegger (mais Lorenz aussi

\footnotetext{
${ }^{10}$ C'est Uexküll qu'on crédite pourtant le plus souvent d'avoir inauguré une pensée " généreuse " de l'animal-sujet s'ouvrant un milieu de significations - pensée susceptible de faire pièce au mécanisme -, et qui a lui-même, à l'occasion, essayé de donner une représentation figurée du monde de tel ou tel animal.
} 
bien) restent pris dans une conception physicaliste, statique et essentialiste des lois de la vie, qui ne fait pas assez droit à l'historicité ouverte et à la contingence radicale du vivant. À l'inverse, Canguilhem proposera d'opposer à un système de lois l'idée d'un d'ordre de la vie - ordre fait de compositions de forces, de compromis toujours instables et précaires -, et prendra à rebours l'idée d'une perfection du vivant, posant que les formes animales ou végétales méritent d'être critiquées tout autant que d'être admirées, affirmant que les réussites n'y sont que des échecs retardés et les échecs des réussites avortées, soulignant la présence d'organes inutiles, de monstruosités ou de comportements aberrants, qui constituent autant de lieux $\mathrm{d}^{\prime}$ invention et d'expérimentation vitale dont la valeur ne s'attestera ou ne s'infirmera toujours qu'a posteriori ${ }^{11}$.

Revenons au projet de construire un analogon de l'expérience et du monde d'un animal. Nous avons affirmé précédemment que c'est ce que Heidegger tente dans son cours en s'inspirant des travaux d'Uexküll, et pensons que c'est vraiment ce qu'il fait pratiquement. Pourtant - en laissant ici de côté la question brûlante de savoir sur quels travaux empiriques se fonder pour accomplir cette reconstruction, et comment les interpréter -, il semble que Heidegger ait aussi proposé une autre voie, qu'il thématise mais pratique peu dans son cours. Cette voie, c'est celle d'une «transposition » [Sichversetzen] pensée non pas comme un "se mettre à la place de », mais comme un "accompagner" [Mitgehen], au sens d'un cheminer ensemble (en s'ouvrant la possibilité d'accéder à l'autre à la faveur de ce cheminement ${ }^{12}$ ) à travers un espace commun - espace dont le caractère commun est pourtant, dans le cas de «l'animal $»^{13}$, mais déjà même dans le cas de l'homme ${ }^{14}$, éminemment problématique. Nous sollicitons peut-être un peu le texte de Heidegger (qui semble parfois orienté téléologiquement par ses conclusions sur la pauvreté en monde ${ }^{15}$ ), parce qu'il touche et permet de penser un problème essentiel, qui croise l'ontologie et la politique : celui de la définition et/ou de la composition du commun. Un détour par le travail d'Etienne Balibar nous permettra d'articuler cette question au problème de la ou des

\footnotetext{
${ }^{11}$ Cf. Georges Canguilhem, La connaissance de la vie, Paris, Vrin, 2003, p. $204-207$ [ Le normal et le pathologique»] et p. 152 [« Machine et organisme»]; Georges Canguilhem et al., Du développement à l'évolution au XIXe siècle, Paris, PUF, "Quadrige», 2003, p. 78 ["Subordination du concept d'épigenèse au concept d'évolution des espèces : Darwin (1859)»].

12 GA 29/30, p. 296-297 (trad. p. 299-300).

${ }^{13}$ GA 29/30, p. 299 (trad. p. 302).

14 GA 29/30, p. 301-302 (trad. p. 304).

15 «L'animal» offre une sphère possible de transposition MAIS refuse l'accompagnement cf. par ex. GA 29/30, p. 308 (trad. p. 311).
} 
différences anthropologiques, et de prolonger les intuitions de Heidegger dans une direction qui n'a plus grand-chose à voir avec la phénoménologie, mais qui entretient d'une certaine façon un dialogue silencieux avec elle ${ }^{16}$.

Le contexte problématique dans lequel Balibar mobilise le concept de différences anthropologiques, est celui de la définition et de l'accès à la sphère de l'universel, que nous identifierons ici (en simplifiant quelque peu la complexité du texte ${ }^{17}$ ) comme sphère du commun. Ce contexte est aussi celui d'une anthropologie politique, qui refuse de penser l'anthropogenèse en dehors de son inscription dans des conjonctures historiques et politiques, et qui est thématisée par contraste avec une anthropologia perennis. L'idée de Balibar est qu'au sein de l'espace ou de l'époque qu'ouvre la Déclaration des droits de l'homme et $d u$ citoyen, l'exclusion de la sphère de la citoyenneté (qui est à la fois celle de l'universel et du commun) passe par une exclusion de la sphère de l'humanité. Or Balibar pose que la figure de l'humanité ne peut, à une époque donnée, se définir sans poser des bords ou des limites, qui constituent autant de différences à la fois externes (différence homme/animal) et internes (différence homme/femme, adulte/ enfant, bien-portant/malade, etc.). La tension paradoxale qui mobilise sa réflexion sur les différences anthropologiques, c'est que l'ensemble de ces différences, qui fonctionnent comme autant d'opérateurs de production d'une sous-humanité, comme vecteurs d'exclusion de la sphère de l'humanité pleine et entière (donc par contrecoup de la sphère de l'universalité et du commun), sont également ce qui - à la faveur de mouvements politiques d'émancipation qui s'appuient sur une mise en évidence des contradictions de l'universalisme ${ }^{18}$ - va permettre à l'universel de se réaliser véritablement.

La différence est donc à la fois ce qui fait obstacle à l'universalité, et ce qui en permet la réalisation. Balibar ajoute que ces différences anthropologiques sont à la fois incontournables (on ne peut imaginer l'humain sans l'existence de ces partages), indéniables (données presque perceptivement, et donc susceptibles d'une phénoménologie), ET pourtant indéfinissables, inassignables une fois pour toutes (les partages

\footnotetext{
${ }^{16}$ L'expression «différences anthropologiques", écrite au pluriel, est un concept qui traverse plusieurs textes d'Etienne Balibar, et fait l'objet d'un examen approfondi dans la section «Malêtre du sujet : universalité bourgeoise et différences anthropologiques ", qui clôt le volume Citoyen Sujet et autres essais d'anthropologie philosophique, Paris, PUF, «Pratiques théoriques », 2011, p. 463515.

${ }^{17}$ Cf. par ex. Citoyen Sujet, p. 30.

${ }^{18}$ En mobilisant des énoncés ou des argumentations que Balibar range sous la catégorie de synecdoque de l'universel, par ex. : «Les femmes sont-elles des hommes comme les autres? ».
} 
ou différences en question ne relèvent pas d'une essence qui pourrait être fixée une bonne fois, mais sont en quelque sorte virtuellement flottants, toujours renégociables). Cette approche balibarienne de la question des différences anthropologiques, et le cadre dialectique ou aporétique dans lequel elle les inscrit, permettent de soulever plusieurs questions importantes.

Si les différences anthropologiques sont à la fois incontournables/indéniables et indéfinissables/inassignables, quelle est la responsabilité d'une anthropologie phénoménologique, c'est-à-dire d'un discours qui fonde sur une description phénoménologique la définition de l'essence de l'humanité, et l'assignation du lieu des différences anthropologiques (que celles-ci soient "externes»- homme/ « animal » - ou « internes » - normal/pathologique, masculin/féminin, etc.)? Le discours de l'anthropologie phénoménologique ne fonctionne-t-il pas alors de manière potentiellement ambivalente, dans un registre où la description - somme toute légitime - d'un donné indéniable devient inséparable d'une ratification/prescription - somme toute contestable - de lignes de partage historiquement contingentes, ratification qui du même coup fait virtuellement obstacle à leur déplacement éventuel ? Ce que la prise au sérieux du cadre ou de la structure esquissée par Balibar permet, c'est d'adresser à la phénoménologie une question plus polie et respectueuse que le reproche intégralement constructiviste qui dirait: "Vous prétendez décrire, mais au fond vous ne faites que produire/prescrire l'objet de votre description ».

La différence anthropologique proprement dite (le partage homme/« animal ») ne fait pas l'objet d'un développement exprès dans le texte de Balibar. On pourrait pourtant risquer l'hypothèse qu'elle constitue un cas à la fois limite et exemplaire du processus de position de l'universel fonctionnant suivant un double mouvement (toujours renégociable) d'inclusion et d'exclusion - ce qui est exclu pouvant à la faveur de mobilisations politiques précisément se présenter et s'argumenter comme étant ce dont la prise en compte ou l'inclusion permettra à l'universel de se réaliser véritablement. Si des lignes de partage se tracent en permanence au sein de l'humanité, qui la divisent tout en la mettant en relation avec elle-même, de semblables lignes se tracent également au sein de l'ensemble plus vaste que forment les vivants humains et non humains. C'est ainsi qu'à rebours d'une position moralisatrice qui prônerait le respect de la vie en général, Donna Haraway avance une position " non-innocente » qui pose qu'on ne peut vivre sans tuer d'autres formes de vie, et que la question importante n'est pas celle de la mort, mais celle de savoir 
quelles vies sont désignées comme «tuables » ${ }^{19}$. Dans le même monde moderne coïncident ainsi les vies killable des bêtes d'abattoirs et les vie non-killable des animaux de compagnie. Ce partage - qui sera peut-être amené à bouger dans les décennies qui viennent, non pas massivement, mais dans son détail, au cas par cas - n'est pas sans rappeler le partage (opéré au sein même de l'humanité) que pointe Judith Butler, dans ses réflexions de l'après 11 septembre, entre les vies dignes d'être pleurées (et qui s'attestent par là comme proprement humaines) et les autres. Ce que nous proposons, en rapprochant ici la réflexion d'Haraway de celle de Butler, $c^{\prime}$ est de faire l'hypothèse que LA différence anthropologique (entendue comme partage entre l'homme et «l'animal»), en tant qu'en elle se pensent et se négocient les figures de l'altérité maximale, constitue peut-être le lieu par excellence d'invention et de création pour une politique qui tente d'articuler différence et constitution de l'universel ou du commun.

On pourrait tenter de donner corps à cette hypothèse en essayant de retourner ou de faire travailler l'analogie - abondamment thématisée depuis un demi-siècle - entre les différentes formes historiques d'exclusion de l'humanité opérées au sein même de celle-ci (soushumanité des femmes, des enfants, des sauvages, des malades mentaux, etc.) et l'exclusion philosophique radicale de «l'animal » dans sa participation virtuelle à tout ce qui pourrait ressembler de près ou de loin à un propre de l'homme. Si l'animalité a fonctionné historiquement comme matrice pour la pensée des différences anthropologiques "internes", elle n'a pu le faire que parce qu'un "air de famille » affectait les modes de perception et de pensée d'époques pas si lointaines, qui rapprochaient par exemple le mutisme de l'enfant ou du fou de celui de l'animal. On sait ce que cet « air de famille » a pu avoir comme conséquences politiques, et l'on pourrait même à cet égard créditer le travail d'une anthropologie phénoménologique attentive à distinguer radicalement le vécu perceptif de l'infans humain de celui de n'importe quel animal adulte - de la vertu d'empêcher ce genre d'analogie funeste. On peut pourtant aussi envisager les choses par un autre bout, en considérant que les renégociations « expérimentales » de la ligne de partage qui nous sépare et nous relie aux vivants non-humains (- par ex. lorsque des animaux de laboratoire parviennent à s'opposer à un dispositif expérimental peu courtois, ou lorsque des espèces qui partagent un même territoire apprennent à faire des compromis qui permettent le vivre-ensemble

\footnotetext{
${ }^{19}$ Emilie Hache, «If I have a dog, my dog has a human », in Elsa Dorlin \& Eva Rodriguez, Penser avec Donna Haraway, Paris, PUF, « Actuel Marx Confrontation », 2012, p. 97.
} 
sous une forme moins violente ou plus civilisée) constituent un laboratoire qui nous propose des schèmes de mobilisation ou d'argumentation susceptibles d'être employés dans une renégociation politique des différences anthropologiques internes. Ce que nous apporte le travail à la fois scientifique et politique autour de l'animalité, c'est par exemple un début de réponse à la question : "Comment construire du commun, comment négocier le commun, avec des êtres qui "d'évidence" ne parlent pas ?".

Si l'on prend au sérieux la topique balibarienne, on est donc en droit de reformuler la question précédemment adressée à l'anthropologie phénoménologique, en l'appliquant tout particulièrement à sa description du partage ou de la différence homme/« animal», en tant que cette différence constitue le lieu où s'affrontent par excellence l'indéniable (véhiculé jusque dans la langue maternelle, qui pose l'évidence du partage radical entre l'homme et le reste des espèces unifiées en un seul domaine homogène) et l'inassignable (domaine que nous ouvrent les travaux récents en éthologie, ainsi qu'une certaine épistémologie constructiviste). Nous exposerons en guise de conclusion quelques développements récents situés du côté de l'inassignable, en les articulant à une double thèse heideggérienne : celle de l'historicité de l'être, et celle de la dimension performative de la langue qui participe à son avènement. Bon nombre des travaux récents qui gravitent autour de la question de l'animalité en articulant épistémologie et politique partagent en effet ce présupposé d'une historicité de l'être et d'une performativité de la langue. On prendra ici comme exemple le cas de Vinciane Despret, et de l'ensemble des chercheurs (notamment biologistes et éthologues) qu'elle mobilise ou met en valeur dans ses livres.

Nous avons affirmé précédemment qu'un des aspects passionnants de la phénoménologie, lorsqu'elle se confronte au problème de l'animalité, c'est qu'elle ne peut faire l'économie d'une confrontation et d'un dialogue avec les sciences empiriques. Nous avons également fait part de nos réticences à l'égard de la conception intégralement "fonctionnaliste » d'Uexküll (qui est aussi celle de Lorenz et de l'éthologie naissante), qui constitue l'horizon des analyses du cours de 1929-30. Cette conception - dont on pourrait soutenir qu'elle est coextensive au désir de fonder l'éthologie comme discipline scientifique rigoureuse, à la fois munie d'un attirail de lois, et séparée du discours « anthropomorphique » qui caractérise le savoir profane des éleveurs, chasseurs, dresseurs ou naturalistes amateurs - est aujourd'hui discutée, et remise en question à bien des égards. L'un des intérêts du travail de Vinciane Despret est qu'elle suit ou accompagne 
les lieux et les êtres qui contribuent à remettre ce modèle en cause : observations de terrain surprenantes, qui résultent d'un bouleversement de la population observatrice ${ }^{20}$ ou de l'attention portée à des animaux qui « $\mathrm{n}^{\prime}$ avaient pas la cote $»^{21}$, dispositifs expérimentaux plus ingénieux et plus polis à l'égard de leurs objets/sujets d'expérience ${ }^{22}$, mais aussi récente prise au sérieux des savoirs profanes ${ }^{23}$.

Ces bouleversements scientifiques et extrascientifiques, Despret ne les présente pas comme un simple changement de perspective sur une réalité qui n'aurait pas bougé, mais - et nous retrouvons ici la thèse heideggérienne d'une historicité de l'être - comme une modification ontologique : quelque chose se passe dans l'être-avec qui rassemble bêtes et hommes; les chercheurs et les publics profanes changent, mais leurs animaux changent également, et l'on n'est jamais sûr de savoir d'où vient au juste l'initiative. Un deuxième trait caractéristique de ces travaux scientifiques et de ces bouleversements, dans la présentation qu'en offre Despret, c'est l'attention passionnée qui est portée à la dimension performative de la façon dont les humains s'adressent aux animaux - ou l'inverse. Dans cette perspective, l'expérimentation animale n'est plus conçue comme révélation de lois immuables de l'espèce, elle est considérée comme une proposition d'expérience faite à un animal singulier. Cette proposition peut être ou non intéressante. Si elle l'est, elle risque de rendre l'animal intéressant, et avec lui son chercheur.

Ces analyses philosophiques des mutations récentes des rapports entre bêtes et hommes n'ont plus grand rapport avec la phénoménologie. Elles permettent pourtant de donner corps à certaines des propositions les plus audacieuses du cours de 1929-30 : considérer que le changement de langue peut ( $\left.\mathrm{s}^{\prime}\right)$ accompagner $\left(\mathrm{d}^{\prime}\right)$ une transformation de l'existence et du rapport à l'être, et remplacer la volonté de «se mettre à la place » par l'expérience d'un accompagnement qui peut déboucher sur une connaissance de l'autre. Ces analyses, et les travaux dont elles rendent compte, ont abandonné le postulat d'une séparation radicale entre l'homme et l'animal, mais n'aboutissent pourtant ni à une totale indistinction, ni à un moralisme niais. Elles s'intéressent au détail, aux singularités, à la façon dont le monde

\footnotetext{
${ }^{20}$ Ce fut le cas lors de l'arrivée de femmes sur le terrain en primatologie.

${ }^{21}$ Par exemple étudier des moutons (Thelma Rowell) ou des corbeaux (Benrd Heinrich) avec la même générosité que celle qu'on accorderait aux singes supérieurs.

22 Étudier le bien-être plutôt que la souffrance nous oblige par exemple à ralentir considérablement.

${ }^{23}$ Par exemple le savoir des éleveurs - longtemps disqualifié par celui des zootechniciens - ou celui des dresseurs.
} 
commun n'est pas un donné mais plutôt le résultat d'un compromis construction de points de vue inédits issus de rencontres interspécifiques, solution toujours précaire à des problèmes dont les humains n'ont plus le monopole de définir les conditions.

Les développements récents de la recherche et de la réflexion sur la question animale pourraient donc conduire à abandonner l'idée $\mathrm{d}^{\prime}$ une ontologie à foyers multiples fondée sur le désir de « se mettre à la place » de l'animal, et à la remplacer par le projet d'une métaphysique expérimentale (Latour) fondée sur l'idée de point de vue composé (Haraway ${ }^{24}$ ) institué à la faveur d'un apprentissage/agencement collectif et interspécifique (Despret). Si le cours de 1929-30 est si intéressant, c'est peut-être en ce qu'il ouvre ces deux voies, opposées et concurrentes: celle du «se mettre à la place de l'animal », que l'éthologie d'Uexküll conduit selon nous à l'échec, et celle du " cheminer-avec, en découvrant l'autre, et en négociant la sphère problématique du commun »- voie qu'il ne fraie pas, mais dont il indique la direction, et que les scientifiques et philosophes qui prennent au sérieux la question des relations entre bêtes et hommes, avec pragmatisme et sans moralisme, travaillent aujourd'hui à explorer.

\footnotetext{
${ }^{24} \mathrm{Cf}$. Sur ce point Benedikte Zitouni, «With whose blood were my eyes crafted ? Les savoirs situés comme la proposition d'une autre objectivité ", in Penser avec Donna Haraway, op. cit., notamment p. 56-57. Haraway : "Le moi connaissant est partiel dans toutes ses manifestations, jamais fini, ni entier, ni simplement là, ni originel ; il est toujours composé et suturé de manière imparfaite, et donc capable de s'associer avec un autre, pour voir avec lui, sans prétendre être l'autre ». ("Savoirs situés. La question de la science dans le féminisme et le privilège de la perspective partielle»).
} 